\title{
Análise da correlação entre hipovitaminose $D$, dor crônica e depressão em idosos
}

\author{
Analysis of the correlation between hypovitaminosis $D$, chronic pain and depression in the \\ elderly
}

\section{Análisis de la correlación entre hipovitaminosis $D$, dolor crónico y depresión en ancianos}

Camila Araujo Oliveira ${ }^{1 *}$, Silvia Christiane de Jesus Paes ${ }^{1}$, Marina Maria Guimarães Borges ${ }^{1}$, Tellyson Araujo Furtado², Artur dos Santos Soares², Thaís Vieira Tangerino², Rafaela Antônio de Bastos Ribeiro².

\section{RESUMO}

Objetivo: Caracterizar dor crônica e sua associação com hipovitaminose $\mathrm{D}$ e sintomas depressivos em idosos atendidos em um Ambulatório. Métodos: Aplicou-se um questionário acerca da presença ou não de queixas álgicas, assim como sobre sintomas de humor associados. Para ambos os grupos, empregou-se a "Escala de Depressão Geriátrica, utilizou-se o "Questionário de Dor Neuropática em 4 Questões" (DN4) nos pacientes com dor. Resultados: Embora não apresentando significância estatística $(p>0,05)$, observou-se que a maioria dos pacientes deste estudo se tratava de mulheres idosas $(78,2 \%)$, as quais representaram $80 \%$ do grupo com dor. Neste, detectou-se que a dor nociceptiva, em relação à neuropática, foi predominante $(60 \%$ vs. $40 \%$, respectivamente). Ainda, baixos níveis de vitamina $D$ foram encontrados em metade dos pacientes com dor nociceptiva. Em comparação ao grupo controle, houve predominância de pacientes depressivos no grupo com dor (28\% vs. $40 \%$, respectivamente) - e, nestes, a dor neuropática prevaleceu (58,3\%). Conclusão: As características clínico-epidemiológicas dos pacientes e a possível associação entre hipovitaminose $\mathrm{D}$, dor crônica e depressão pôde ser, de algum modo, estabelecida neste estudo - ainda que não tenhamos atingido um $p$-valor significativo $(p<0,05)$ e diante de uma casuística a qual se demonstrou insuficiente para desfechos mais fidedignos.

Palavras-chave: Dor crônica, Deficiência de vitamina D, Depressão.

\section{ABSTRACT}

Objective: To characterize chronic pain and its association with hypovitaminosis $D$ and depressive symptoms in the elderly treated at an outpatient clinic. Methods: A questionnaire was applied about the presence or absence of pain complaints, as well as about associated mood symptoms. For both groups, the "Geriatric Depression Scale, the" Neuropathic Pain Questionnaire in 4 Questions "(DN4) was used in patients with pain. Results: Although not showing statistical significance ( $p>0.05)$, it was observed that most patients in this study were elderly women $(78.2 \%)$, who represented $80 \%$ of the group with pain. In this study, nociceptive pain, in relation to neuropathic pain, was found to be predominant ( $60 \%$ vs. $40 \%$, respectively). In addition, low levels of vitamin D were found in half of patients with nociceptive pain. Compared to the control group, there was a predominance of depressive patients in the pain group ( $28 \%$ vs. $40 \%$, respectively) - and in these, neuropathic pain prevailed (58.3\%). Conclusion: The clinical-epidemiological characteristics of the patients and the possible association between hypovitaminosis $\mathrm{D}$, chronic pain and depression could, in some way, be established in this study - even though we did not reach a significant $p$-value $(p<0.05)$ and in the face of a series which proved insufficient for more reliable outcomes.

Keywords: Vitamina D deficiency, Chronic pain, Depression.

\section{RESUMEN}

Objetivo: Caracterizar el dolor crónico y su asociación con la hipovitaminosis $\mathrm{D}$ y los síntomas depresivos en los ancianos tratados en una clínica ambulatoria. Métodos: Se aplicó un cuestionario sobre la presencia o ausencia de quejas de dolor, así como sobre los síntomas del estado de ánimo asociados. Para ambos grupos, la "Escala de depresión geriátrica", el "Cuestionario de dolor neuropático en 4 preguntas" (DN4) se

\footnotetext{
${ }^{1}$ Universidade Federal do Pará (UFPA), Belém - PA. * E-mail: oliveiracamila.md@gmail.com

${ }^{2}$ Centro Universitário Metropolitano da Amazônia (UNIFAMAZ), Belém - PA.
}

SUBMETIDO EM: 7/2020

ACEITO EM: 8/2020

PUBLICADO EM: 12/2020 
utilizó en pacientes con dolor. Resultados: Aunque no mostró significación estadística ( $p>0.05)$, se observó que la mayoría de los pacientes en este estudio eran mujeres de edad avanzada (78.2\%), que representaban el $80 \%$ del grupo con dolor. En este estudio, el dolor nociceptivo, en relación con el dolor neuropático, resultó ser predominante (60\% frente a $40 \%$, respectivamente). Además, se encontraron niveles bajos de vitamina $D$ en la mitad de los pacientes con dolor nociceptivo. En comparación con el grupo de control, hubo un predominio de pacientes depresivos en el grupo de dolor (28\% frente a $40 \%$, respectivamente), y en estos prevaleció el dolor neuropático (58.3\%). Conclusión: Las características clínico-epidemiológicas de los pacientes y la posible asociación entre la hipovitaminosis $\mathrm{D}$, el dolor crónico y la depresión podrían, de alguna manera, establecerse en este estudio, a pesar de que no alcanzamos un valor $p$ significativo $(p<0.05)$ y frente a una serie que resultó insuficiente para obtener resultados más confiables.

Palabras clave: Deficiencia de vitamina D, Dolor crónica, Depresión.

\section{INTRODUÇÃO}

A população mundial está em processo de envelhecimento e, de acordo com a Organização das Nações Unidas (ONU), a população com idade igual ou superior a 60 anos era equivalente a 962 milhões em 2017, mais de duas vezes do que em 1980, quando o total correspondia a 382 milhões. A tendência é que a população dobre novamente até o ano 2050, com valores projetados de aproximadamente 2,1 bilhões de idosos no mundo todo (UNITED NATIONS, DEPARTMENT OF ECONOMIC AND SOCIAL AFFAIRS, 2017).

O Brasil vem acompanhando a tendência mundial e, durante mais de 500 anos, o país teve uma estrutura etária rejuvenescida, a qual começou a mudar no século XX a XXI quando a população migrou do campo para a cidade e a nova estrutura familiar exigia menos filhos (MIRANDA D, et al., 2016).

Em 2018, o Instituto Brasileiro de Geografia e Estatística (IBGE) ratificou a mudança na pirâmide populacional, antes de base larga (rejuvenescida), para uma pirâmide de base e topo estreitos. Isto, condizente, portanto, com um país envelhecido, no qual a proporção de pessoas idosas aumentou e de jovens reduziu (IBGE, 2018).

Ainda, segundo o IBGE, o Brasil se tornará um país mais envelhecido em 2031, quando haverá 42,3 milhões de jovens de 0 a 14 anos e 43, 3 milhões de idosos com 60 anos ou mais (IHU, 2018). Esses números são consequência da redução das taxas de fertilidade e do aumento da expectativa de vida (UNITED NATIONS, DEPARTMENT OF ECONOMIC AND SOCIAL AFFAIRS, 2017).

Em consonância com o aumento da população idosa, a dor crônica representa uma característica comum com $66 \%$ das pessoas com idade superior a 65 anos. Mulheres costumam ser mais afetadas comparado aos homens (CORSI N, et al., 2018). Nos Estados Unidos, por exemplo, cerca de 100 milhões de americanos ou um terço da população, são afetados por esta realidade. Cerca de 25 milhões têm dor crônica moderada a grave que limitam suas atividades e diminui a qualidade de vida (SORGE RE, et al., 2017).

A dor crônica, por ser um problema clínico complexo, exige uma abordagem individualizada e multifacetada. Estende-se por uma infinidade de condições, com apresentações e etiologias variadas. Pessoas que vivem com este sintoma são frequentemente agrupadas em uma única categoria, com abordagens de tratamento muitas vezes generalizados. Embora a dor seja um fenômeno dinâmico - que se intensifica e diminui ao longo do tempo, muitas vezes, é vista e gerenciada mediante uma abordagem estática (SORGE RE, et al., 2017).

$\mathrm{Na}$ tentativa de buscar terapêuticas mais eficazes e com menos efeitos adversos para dor crônica em idosos, especialmente considerando indivíduos com dores neuropática e musculoesquelética, importantes estudos relacionam a vitamina $D$ a quadros dolorosos, o que poderia propor sua suplementação como uma alternativa analgésica.

Uma metanálise contendo 71 artigos identificou que as concentrações de vitamina $D$ demonstraram-se em níveis menores nos idosos portadores de osteoartrite, dor crônica difusa e dor muscular (WU Z, et al., 2018).O objetivo do presente artigo foi caracterizar a dor crônica e sua associação com hipovitaminose $D$ e sintomas depressivos em idosos atendidos em um Ambulatório de Geriatria. 


\section{MÉTODOS}

Trata-se de um estudo descritivo, observacional e transversal. Esta pesquisa foi realizada em um ambulatório de geriatria no período de janeiro a maio de 2020. A casuística do presente trabalho comporta pacientes longevos a partir dos 60 anos, de ambos os sexos, residentes na comunidade, atendidos no Serviço de Geriatria e com, pelo menos, duas comorbidades.

Os participantes da pesquisa foram divididos em dois grupos: "Grupo Dor" (GD - Grupo 1), composto por indivíduos com diagnóstico de dor crônica, não oncológica, osteomuscular ou neuropática; "Grupo Controle" (GC - Grupo 2), constituído por indivíduos sem diagnóstico de dor crônica e em tratamento de outra patologias. Em ambos os grupos, os indivíduos foram recrutados de forma aleatória no Ambulatório de Geriatria e a amostra coletada foi equivalente a 55 pacientes.

Neste estudo, foi utilizada a definição de dor crônica estipulada pela "Internacional Association for the Study of Pain" (IASP), de modo que os pacientes que foram incluídos nesta pesquisa para fazer parte do GD são idosos com 60 anos ou mais com habilidade para deambular independentemente, capacidade de responder a todos os questionários e, também, disponibilizar amostra de sangue para análise. Ademais, para composição do GC, considerou-se pacientes idosos sem queixas álgicas.

Foram excluídos desta pesquisa todos aqueles que apresentaram o diagnóstico de hipotireoidismo descompensando, transtorno cognitivo maior em fase moderada a avançada, aplicando-se, neste caso, a escala "Functional Assessment Staging" (FAST), história de insuficiências renal ou hepática, bem como história de dor relacionada ao câncer e ao tratamento de câncer. Também, foram excluídos desta pesquisa os idosos já em uso de vitamina $\mathrm{D}$ em doses superiores a 1000Ul diárias.

Aplicou-se uma Ficha Epidemiológica para coleta de dados, a qual inclui informações como sexo, idade, raça, estado civil e escolaridade dos indivíduos. Os pacientes foram avaliados individualmente e a aquisição dos dados feita no momento da consulta foi após os devidos esclarecimentos sobre o estudo e mediante a assinatura do "Termo de Consentimento Livre e Esclarecido" (TCLE) pelo paciente.

Aos pacientes pertencentes ao GD, foram realizadas perguntas a fim de caracterizar a sua dor quanto ao tipo, à intensidade e aos sintomas de humor associados. Ademais, no intuito de mensurar a intensidade da dor, foi utilizada a Escala Verbal Numérica, que avalia a dor em uma escala de intensidade variável entre 0 a 10 - cuja pontuação 0 representa "nenhuma dor" e 10 indica "a pior dor".

Para a avaliação de possível repercussão da dor sobre o humor do idoso, foi aplicada a "Escala de Depressão Geriátrica" (GDS, do inglês "Geriatric Depression Scale") na sua versão contendo 15 itens, validada no Brasil e amplamente utilizada como instrumento de rastreio de depressão em pacientes idosos. Trata-se de um teste para detecção de sintomas depressivos no idoso, com 15 perguntas (GDS-15) negativas/afirmativas onde o resultado igual 5 ou mais pontos é indicativo da existência de sintomas depressivos (STOPPE J, et al., 1994).

A GDS-15 também foi aplicada ao grupo controle para fins de comparações estatísticas e de avaliação da frequência de transtornos de humor entre os idosos com dor crônica e aqueles sem esta queixa. Além disso, foi utilizado o "Questionário de Dor Neuropática em 4 Questões" (DN4 - Douleur Neuropathique 4 Questions) nos pacientes que se enquadraram no GD.

Aos dois grupos, foi solicitada a dosagem de $25-\mathrm{OH}$-vitamina $\mathrm{D}$ sérica para fins de comparação. Os níveis de vitamina $D$ considerados na faixa de referência normal variam entre 30 a $100 \mathrm{ng} / \mathrm{ml}$ e são caracterizados como insuficientes quando se encontram entre 21 e $29 \mathrm{ng} / \mathrm{ml}$ e deficientes quando menor que $20 \mathrm{ng} / \mathrm{ml}$ (HOLICK MF, et al., 2011).

A amostra sanguínea de cada participante da pesquisa foi coletada pelo Laboratório do HUJBB e encaminhada ao Laboratório Ruth Brazão, ressaltando-se que a solicitação deste exame já faz parte da rotina admissional do paciente no Ambulatório de Geriatria. Por fim, todas as informações obtidas durante a coleta de dados foram transferidas e agrupadas em planilhas eletrônicas usando-se o software Microsoft Excel 2013. 
Este estudo foi realizado seguindo os preceitos da "Declaração de Helsinque", do "Código de Nuremberg", e das "Normas de Pesquisa Envolvendo Seres Humanos" (Res. CNS 466/12) do Conselho Nacional de Saúde (CNS), mediante aprovação do projeto de pesquisa pelo Comitê de Ética em Pesquisa (CEP) sob número de parecer 3.960.138. Os sujeitos da pesquisa ou responsáveis somente tiveram os dados inclusos na pesquisa por meio da assinatura do TCLE após os devidos esclarecimentos acerca do estudo.

Os dados foram coletados e catalogados em planilha no programa Microsoft Excel 2010. Os gráficos e tabelas foram construídos com as ferramentas disponíveis nos softwares Microsoft Word, Excel e Bioestat 5.5. Os testes foram executados com o auxílio do software BioEstat 5.5. As variáveis quantitativas foram submetidas à análise descritiva (mínimo, máximo, média e desvio padrão) e as variáveis qualitativas descritas por frequência e percentagem. O Teste $G$ (para duas amostras independentes) foi utilizado na comparação dos dados sociodemográficos e clínicos entre grupo dor e grupo controle, ou entre dois grupos dor, também utilizado para comparação dos resultados de GDS, níveis séricos de vitamina e IMC entre o grupo dor e o grupo controle, ou entre dois grupos dor. Os resultados com $p \leq 0,05$ (bilateral) foram considerados estatisticamente significativos.

\section{RESULTADOS}

Foram incluídos neste estudo 55 pacientes, sendo 43 (78\%) do sexo feminino e 12 (22\%) do sexo masculino. Do total, 30 pacientes (55\%) possuíam dor crônica e 25 (45\%), alocados ao grupo controle, não possuíam. As idades variaram de 63 a 92 anos, sendo que no grupo Dor, mais da metade possuía de 70 a 79 anos. A etnia era predominantemente parda nos dois grupos, a maior parte era de aposentados e o estado civil, predominantemente casados. A maioria dos indivíduos com dor possuíam ensino fundamental (completo ou incompleto) e os sem dor, ensino médio. Observa-se que os grupos Dor e Controle não diferiram significativamente quanto às características sociodemográficas avaliadas (sexo, idade, etnia, profissão, estado civil e escolaridade) (Tabela 1).

Tabela 1 - Características sociodemográficas e associação à dor crônica, dos pacientes atendidos no Serviço de Geriatria e Gerontologia.

\begin{tabular}{|c|c|c|c|c|}
\hline Variável & Geral & Com Dor $(n=30)$ & Sem Dor $(n=25)$ & p-valor \\
\hline Sexo & & & & 0,976 \\
\hline Feminino & $43(78,2)$ & $24(80,0)$ & $19(76,0)$ & \\
\hline Masculino & $12(21,8)$ & $6(20,0)$ & $6(24,0)$ & \\
\hline Idade (anos) & & & & 0,175 \\
\hline 63 a 69 & $17(30,9)$ & $9(30,0)$ & $8(32,0)$ & \\
\hline 70 a 79 & $24(43,6)$ & $16(53,3)$ & $8(32,0)$ & \\
\hline 80 a 92 & $14(25,5)$ & $5(16,7)$ & $9(36,0)$ & \\
\hline Etnia & & & & 0,153 \\
\hline Branco & $2(3,6)$ & $1(3,3)$ & $1(4,0)$ & \\
\hline Indígena & $1(1,8)$ & $0(0,0)$ & $1(4,0)$ & \\
\hline Negro & $3(5,5)$ & $3(10,0)$ & $0(0,0)$ & \\
\hline Pardo & $49(89,1)$ & $26(86,7)$ & $23(92,0)$ & \\
\hline Profissão & & & & 0,246 \\
\hline Aposentado & $33(60,0)$ & $20(66,7)$ & $13(52,0)$ & \\
\hline Autônomo & $10(18,2)$ & $6(20,0)$ & $4(16,0)$ & \\
\hline Do Lar & $12(21,8)$ & $4(13,3)$ & $8(32,0)$ & \\
\hline Estado Civil & & & & 0,401 \\
\hline Casado & $24(43,6)$ & $14(46,7)$ & $10(40,0)$ & \\
\hline Solteiro & $15(27,3)$ & $10(33,3)$ & $6(24,0)$ & \\
\hline Outros & $16(29,1)$ & $6(20,0)$ & $9(36,0)$ & \\
\hline Escolaridade & & & & 0,200 \\
\hline Ens. Fundamental & $26(47,3)$ & $18(60,0)$ & $8(32,0)$ & \\
\hline Ens. Médio & $19(34,5)$ & $8(26,6)$ & $11(44,0)$ & \\
\hline Ens. Superior & $6(10,9)$ & $2(6,7)$ & $4(16,0)$ & \\
\hline Sem estudo & $4(7,3)$ & $2(6,7)$ & $2(8,0)$ & \\
\hline
\end{tabular}

Legenda: As frequências são representadas como $n(\%)$. As percentagens são relativas ao somatório da coluna. Em todos os casos foi utilizado o teste $\mathrm{G}$.

Fonte: Oliveira CA, et al., 2020. 
Em seguida, avaliou-se a associação entre os grupos Dor e Controle quanto ao GDS e à classificação de nível de vitamina D. Observa-se que $40 \%$ dos pacientes no grupo Dor eram depressivos segundo o GDS, enquanto no grupo Controle, $28 \%$ também o eram. Apesar de superior a proporção de depressivos no grupo Dor, esta diferença não foi significativa $(p=0,516)$. Quanto ao nível de vitamina $D$, observa-se que mais da metade (58\%) dos pacientes tinham níveis normais. O número de pacientes com nível insuficiente de vitamina D foi um pouco maior no grupo Dor ( $40 \%$ versus $20 \%$ ), mas essa proporção não foi significante. Ao unirmos os níveis insuficiente e deficiente em um nível "baixo", o p-valor continua não significativo (Tabela 2).

Tabela 2 - Perfil depressivo, níveis de vitamina $D$ e associação à dor crônica em pacientes atendidos no Serviço de Geriatria e Gerontologia.

\begin{tabular}{ccccc}
\hline Variável & Geral & Com Dor $(\mathbf{n}=\mathbf{3 0})$ & Sem Dor $(\mathbf{n}=\mathbf{2 5})$ & p-valor \\
\hline Escore GDS & & & & 0,516 \\
\hline Depressivo & $19(34,5)$ & $12(40,0)$ & $7(28,0)$ & \\
Normal & $36(65,5)$ & $18(60,0)$ & $18(72,0)$ & \\
\hline Nível de Vitamina D & & & & 0,267 \\
\hline Deficiente & $6(10,9)$ & $3(10,0)$ & $3(12,0)$ & \\
Insuficiente & $17(30,9)$ & $12(40,0)$ & $5(20,0)$ & \\
Normal & $32(58,2)$ & $15(50,0)$ & $17(68,0)$ & 0,282 \\
\hline Nível de Vitamina D & & & \\
\hline Baixo & $23(41,8)$ & $15(50,0)$ & $8(32,0)$ & \\
Normal & $32(58,2)$ & $15(50,0)$ & $17(68,0)$ & \\
\hline
\end{tabular}

Legenda: As frequências são representadas como $\mathrm{n}$ (\%). As percentagens são relativas ao somatório da coluna. Em todos os casos foi utilizado o teste $\mathrm{G}$.

Fonte: Oliveira CA, et al., 2020.

São comparados apenas os pacientes do grupo Dor, conforme o tipo de dor (neuropática ou nociceptiva). Entre os pacientes com dor Neuropática, a maioria (58\%) era depressivo, enquanto que entre os pacientes com dor Nociceptiva, a maioria era normal (72\%). Quanto ao nível de vitamina D, as proporções de deficiência, insuficiência ou normal entre os dois grupos foram bem próximas, similar ao unir os grupos deficiente e insuficiente em um grupo "baixo nível de vitamina D". Em todos os casos não houve significância (Tabela 3).

Tabela 3 - Perfil depressivo, níveis de vitamina D e associação ao tipo de dor crônica nos pacientes atendidos no Serviço de Geriatria e Gerontologia.

\begin{tabular}{ccccc}
\hline Variável & Geral & Dor Neuropática (n=12) & Dor Nociceptiva (n=18) & p-valor \\
\hline Escore GDS & & & & 0,196 \\
\hline Depressivo & $12(40,0)$ & $7(58,3)$ & $5(27,8)$ & \\
Normal & $18(60,0)$ & $5(41,7)$ & $13(72,2)$ & \\
\hline Nível de Vitamina D & & & & 0,965 \\
\hline Deficiente & $3(10,0)$ & $1(8,3)$ & $7(11,1)$ & \\
Insuficiente & $12(40,0)$ & $5(41,7)$ & $7(38,9)$ & \\
Normal & $15(50,0)$ & $6(50,0)$ & $9(50,0)$ & 0,709 \\
\hline Nível de Vitamina D & & & & \\
\hline Baixo & $15(50,0)$ & $6(50,0)$ & $9(50,0)$ & \\
Normal & $15(50,0)$ & $6(50,0)$ & $9(50,0)$ & \\
\hline
\end{tabular}

Legenda: As frequências são representadas como $\mathrm{n}(\%)$. As percentagens são relativas ao somatório da coluna. Em todos os casos foi utilizado o teste $\mathrm{G}$.

Fonte: Oliveira CA, et al., 2020.

Apenas o grupo Dor Neuropática é comparado ao grupo Controle. Quanto ao GDS, a maioria (58\%) do grupo neuropático era depressivo, enquanto a maioria $(72 \%)$ do grupo controle era normal. Quanto à vitamina D, metade do grupo neuropático tinha nível baixo de vitamina e metade tinha nível normal, enquanto no grupo sem dor a maioria era normal. Nestes casos sem significância estatística. Encontrou-se uma associação significativa da presença de dor neuropática em relação à etnia $(p=0,035)$. Neste caso, o número de negros com dor neuropática foi estatisticamente superior ao esperado ao acaso (Tabela 4). 
Tabela 4 - Comparação entre o perfil depressivo e os níveis de vitamina D do grupo Dor Neuropática em relação ao grupo controle, dos pacientes atendidos no Serviço de Geriatria e Gerontologia.

\begin{tabular}{ccccc}
\hline Variável & Geral & Dor Neuropática $(\mathbf{n = 1 2})$ & Sem Dor $(\mathbf{n = 2 5})$ & p-valor \\
\hline Escore GDS & & & & 0,159 \\
\hline Depressivo & $14(37,8)$ & $7(58,3)$ & $7(28,0)$ & \\
Normal & $23(62,2)$ & $5(41,7)$ & $18(72,0)$ & 0,394 \\
\hline Nível de Vitamina D & & & $3(12,0)$ & \\
\hline Deficiente & $4(10,8)$ & $1(8,3)$ & $5(20,0)$ & \\
Insuficiente & $10(27,0)$ & $5(41,7)$ & $17(68,0)$ & 0,489 \\
Normal & $23(62,2)$ & $6(50,0)$ & $8(32,0)$ & \\
\hline Nível de Vitamina D & & & $17(68,0)$ & 0,035 \\
\hline Baixo & $14(37,8)$ & $6(50,0)$ & & \\
Normal & $23(62,2)$ & $6(50,0)$ & $1(4,0)$ & \\
\hline Etnia & & & $1(4,0)$ & \\
\hline Branco & $1(2,7)$ & $0(0,0)$ & $0(0,0)^{*}$ & \\
Indígena & $1(2,7)$ & $0(0,0)$ & $23(92,0)$ & \\
Negro & $3(8,1)$ & $3(25,0) \dagger$ & $9(75,0)$ & \\
Pardo & $32(86,5)$ & &
\end{tabular}

Legenda: As frequências são representadas como $\mathrm{n}(\%)$. As percentagens são relativas ao somatório da coluna. *: A frequência observada foi inferior ao que seria esperado ao acaso. $\dagger$ : A frequência observada foi superior ao esperado. Em todos os casos foi utilizado o teste $G$.

Fonte: Oliveira CA, et al., 2020.

Foi comparado o grupo Dor Nociceptiva ao grupo Controle. Observa-se que foram bem similares a proporção de indivíduos depressivos no grupo nociceptivo e a proporção de indivíduos depressivos no grupo Controle, sem associação significativa (Tabela 5).

Tabela 5 - Comparação entre o perfil depressivo e os níveis de vitamina D do grupo Dor Nociceptiva em relação ao grupo controle, dos pacientes atendidos no Serviço de Geriatria e Gerontologia.

\begin{tabular}{ccccc}
\hline Variável & Geral & Dor Nociceptiva $(\mathbf{n}=\mathbf{1 8})$ & Sem Dor $(\mathbf{n}=\mathbf{2 5})$ & p-valor \\
\hline Escore GDS & & & & 0,743 \\
\hline Depressivo & $12(27,9)$ & $5(27,8)$ & $7(28,0)$ & \\
Normal & $31(72,1)$ & $13(72,2)$ & $18(72,0)$ & \\
\hline Nível de Vitamina D & & & & 0,388 \\
\hline Deficiente & $5(11,6)$ & $2(11,1)$ & $3(12,0)$ & \\
Insuficiente & $12(27,9)$ & $7(38,9)$ & $5(20,0)$ & \\
Normal & $26(60,5)$ & $9(50,0)$ & $17(68,0)$ & 0,382 \\
\hline Nível de Vitamina D & & & & \\
\hline Baixo & $17(39,5)$ & $9(50,0)$ & $8(32,0)$ & \\
Normal & $26(60,5)$ & $9(50,0)$ & $17(68,0)$ &
\end{tabular}

Legenda: As frequências são representadas como $\mathrm{n}(\%)$. As percentagens são relativas ao somatório da coluna. Em todos os casos foi utilizado o teste $\mathrm{G}$.

Fonte: Oliveira CA, et al., 2020.

\section{DISCUSSÃO}

Neste estudo, verificou-se que no grupo com dor crônica a prevalência de idosos do sexo feminino foi maior do que do sexo masculino ( $80 \%$ versus $20 \%$, respectivamente). Fatores biopsicossociais têm grande associação com as diferenças de prevalência e de incidência de dor cônica entre homens e mulheres. Tais distinções vão para além das suas diferenças fisiológicas e hormonais pré-existentes e estão intimamente relacionadas à mediação da dor no sistema nervoso central (SNC) por diferentes células do sistema imunológico (SORGE RE, et al., 2017).

Uma abordagem biopsicossocial é capaz de compreender a dor como uma síndrome multidimensional, na qual existe uma forte interação entre fatores fisiológicos, psicológicos e sociais para o seu desenvolvimento, assim como para sua cronificação e complexidade (EDWARDS RR et al., 2016). Nas mulheres, a prevalência de manifestações dolorosas é maior que o dobro em relação aos homens. 
Além disso, poder ser justificada pelas diferenças biológicas no funcionamento do sistema imune - que estão associadas ao controle e à resposta à dor, bem como aos mecanismos de analgesia - provavelmente subjacentes a esses efeitos verificados (BELFER I, 2017; SORGE RE, et al., 2016).

Em um estudo realizado por Larsson C, et al. (2016) - no qual foram incluídos cerca de 1141 indivíduos idosos com 65 anos ou mais - constatou-se que as mulheres $(63,5 \%)$ relataram significativamente mais dor crônica quando comparadas aos homens (36,5\%), inclusive detectando-se nelas maior frequência de queixas associadas à maior intensidade, duração e acometimento de mais de um local pela dor. Ainda, os hormônios sexuais (como estrógeno, progesterona e testosterona) que apresentam um papel imprescindível e complexo nos processos inflamatórios e na resposta fisiológica à dor (MANSON JE, et al., 2010). Eles também desempenham um importante papel, inclusive, na cronicidade da dor - sendo os mecanismos de resposta à dor distintos entre homens e mulheres (SORGE RE, et al., 2016).

De acordo com estudos mais recentes vêm demonstrando e sugerindo que mecanismos neuroimunes vinculados à modulação da dor representam a etiologia fundamental para as diferenças entre os sexos no que tange sua reação à dor. Também, mencionam que o sexo feminino é mais sensível e menos tolerante a ela (TUFIK S, et al., 2017). Nas mulheres, há maior prevalência de doenças potencialmente inflamatórias por exemplo, artrite e fibromialgia. Sugere-se que as mesmas produzam resposta imune pró-inflamatória aos danos teciduais muito maior do que os homens. Por isso, acabam estando mais suscetíveis à maior inflamação e, consequentemente, à maior intensidade da dor (TUFIK S, et al., 2017).

Ademais, a dor neuropática tem significativa prevalência no sexo feminino por este ser mais suscetível a desenvolvê-la. Nele, há uma frequência maior na ocorrência de doenças autoimunes; também, o seu estrogênio circulante é capaz de elevar a quantidade de citocinas pró-inflamatórias liberadas pelo sistema imunológico em resposta ao estímulo doloroso (TUFIK S, et al., 2017). Em indivíduos portadores de condições médicas cronicamente dolorosas, geralmente, há também um risco significativo de existirem transtornos de humor associados, havendo uma sinergia e potencialização mútua quando há dor e humor deprimido concomitantemente (EDWARDS RR, et al., 2016).

Neste estudo, verificou-se que, apesar de não termos alcançado um $p$-valor significativo $(p=0,516), 40 \%$ dos pacientes do GD são depressivos, em contraste aos $28 \%$ que também o são no GC. Os indivíduos com dor crônica têm, pelo menos, duas vezes mais chances de apresentarem transtorno depressivo e comportamentos suicidas; também de tentarem ou efetivarem o suicídio. Evidências científicas robustas afirmam que a dor crônica é um fator de risco fundamental independente para ele (SHENG J, et al., 2017).

De acordo com pesquisa a dor é comumente associada à depressão na prática clínica, assim como é capaz de ocasionar um importante comprometimento funcional e de exacerbar sintomatologias físicas e psicológicas nesses pacientes. As condições físicas dolorosas crônicas contribuem para maior durabilidade dos sintomas depressivos, além de frequentemente gerarem instabilidade de humor e tristeza aumentada (ISHAK WW, et al., 2018).

Em um estudo, estimou-se que cerca de $20 \%$ dos pacientes com dor crônica apresentou ideação suicida e que as tentativas de suicídio durante a vida variaram entre 5 e $14 \%$ entre eles. Ainda, de acordo, nos sujeitos portadores de dor crônica, houve uma prevalência entre $8 \%$ e $41 \%$ de ideação suicida e entre $14 \%$ e $38 \%$ de tentativas de suicídio (TANG NK, et al., 2018).

Estudos transversais envolvendo pacientes com dor e depressão simultaneamente demonstraram escores menores nas suas medidas de qualidade de vida (QoL) em relação àqueles que apresentavam somente depressão ou ausência de depressão. Além disso, evidenciaram-se correlações significativas entre sintomas depressivos e dor crônica apresentados pelos sujeitos da pesquisa. Isso também foi corroborado por outros estudos os quais demonstraram forte associação entre dor e depressão (ISHAK WW, et al., 2018).

A dor crônica sendo considerada como um estado de estresse orgânico - caracteriza-se como um dos aspectos críticos e determinantes no desenvolvimento do transtorno depressivo. Embora a associação entre ambas as condições médicas permaneça incerta, várias pesquisas demonstraram correlações importantes entre elas, inclusive evidenciando a presença de modificações em mecanismos neurobiológicos - as quais são essenciais à ocorrência de dor crônica e sua indução à depressão (SHENG J, et al., 2018). 
Nesta pesquisa, também verificamos que, embora o $p$-valor não tenha sido significativo $(p=0,267), 0$ percentual de pacientes com níveis insuficientes de vitamina $D$ foi equivalente a $40 \%$ no GD, em contraste aos $20 \%$ encontrado no GC. Além disso, somando-se os percentuais dos pacientes que manifestaram não somente insuficiência (vitamina $D$ sérica $<30 \mathrm{ng} / \mathrm{dL}$ ), mas também deficiência de vitamina $D$ (vitamina $D$ sérica $<20 \mathrm{ng} / \mathrm{dL}$ ), verificamos que $50 \%$ dos pacientes do GD manifestaram taxas circulantes de vitamina $D$ reduzidas, enquanto que somente $32 \%$ dos pacientes no GC manifestou o mesmo.

Há muito tempo, a deficiência e a insuficiência de vitamina $D$ têm sido associadas a distúrbios esqueléticos e, nas últimas décadas, muitos estudos têm sido publicados abordando o papel dos baixos níveis de vitamina D presentes também em distúrbios não-esqueléticos, especialmente em doenças crônicas, como cânceres, doenças autoimunes, HAS, DM, e doenças cardiovasculares. Nos últimos anos, alguns estudos também têm relatado os efeitos positivos da suplementação de vitamina D sobre a dor crônica (CAI, 2019).

Em estudo publicado, o qual foi realizado com 2313 pacientes com idade maior ou igual a 50 anos, verificou-se que, nos pacientes que apresentaram níveis deficientes de vitamina $D(<20 \mathrm{ng} / \mathrm{dL})$, havia um risco aumentado para o desenvolvimento de dor cônica generalizada. Além disso, ressaltou-se que isto poderia estar relacionado também a outras condições médicas, especialmente depressão e obesidade (MCCABE PS, et al., 2016).

Os níveis de vitamina $D$ têm sido inversamente correlacionados com manifestações dolorosas, assim como pesquisas têm demonstrado sua possível ação nos mecanismos regulatórios da dor. Vários estudos também têm indicado que a suplementação de vitamina $D$ seja eficaz na diminuição de queixas álgicas e de distúrbios musculoesqueléticos (TUFIK S, et al., 2017).

Os mecanismos atribuídos à vitamina $\mathrm{D}$ no tratamento da dor incluem efeitos anti-inflamatórios, mediados pela diminuição da liberação de citocinas pró-inflamatórias e de prostaglandinas, principalmente mediante a recente descoberta da inibição da prostaglandina E2 (PGE2) mediada por vitamina D (HELDE-FRANKLING M, et al., 2017).

Em artigo publicado abordou-se quatro casos clínicos de pacientes portadores de dor crônica, os quais foram acompanhados entre seis meses a um ano, e que apresentavam deficiência e insuficiência de vitamina D. No mesmo, percebeu-se significativa melhora (de aproximadamente, 70\%) da dor e dos espasmos musculares após correção dos baixos níveis de vitamina $D$ através de suplementação (50000 Ul de vitamina D por semana, durante 12 semanas) (CAI C, 2019).

Níveis baixos de vitamina $D$ também têm sido vinculados a um maior risco de desenvolvimento de mialgias induzidas por estatinas e, em alguns estudos, demonstrou-se que a sua suplementação com $50000 \mathrm{UI}$ por semana pode ter efeitos protetores contra a mialgia nos pacientes que são tratados com essa classe medicamentosa (CAI C, 2019).

Sabe-se também que a vitamina $\mathrm{D}$ modula o sistema imunológico suprimindo respostas pró-inflamatórias e estimulando respostas anti-inflamatórias (TUFIK S, et al., 2017). Em estudos clínicos, nos quais foi realizada a suplementação da vitamina $D$ nos pacientes com dor crônica, a dor musculoesquelética foi reduzida, juntamente à diminuição sérica dos níveis de citocinas pró-inflamatórias, incluindo a PGE2 (HELDEFRANKLING M, et al., 2017).

Em metanálise realizada verificou-se que a suplementação de vitamina $D$ por um período de três meses diminuiu a ocorrência de dor em pacientes que apresentavam mialgias, artrites e dores musculoesqueléticas crônicas. Sugeriu-se também que a suplementação de vitamina $D$ possa ter papel vital no tratamento da dor crônica (WU Z, et al., 2018).

Mediante o acima exposto, pode-se inferir que há correlação importante entre hipovitaminose $D$, dor crônica e depressão. Sugere-se que níveis reduzidos de vitamina $D$ sejam capazes de desencadear síndromes dolorosas e estas, por sua vez, podem induzir à depressão. Especialmente em indivíduos idosos, a recorrência de queixas álgicas nesse grupo requer, também, sua avaliação psicológica durante suas consultas ambulatoriais. 


\section{CONCLUSÃO}

Neste estudo, não foi atingida significância estatística $(p>0,05)$ diante dos resultados obtidos, bem como a casuística demonstrou-se insuficiente para ratificação ou não de algumas hipóteses as quais, há muito tempo, vêm sendo estudadas no meio científico. Todavia, mesmo diante das limitações para análise dos dados de forma contundente, podemos afirmar que as características clínico-epidemiológicas dos pacientes incluídos nesta pesquisa são compatíveis com as encontradas em outras literaturas vinculadas a este tema. Assim como em muitas evidências científicas recentes, notamos uma prevalência maior de sintomas depressivos e de depressão (GDS-15 $\geq 5$ pontos) nos idosos diagnosticados com dor crônica quando comparados aos controles. Ademais, em relação aos níveis séricos de vitamina $D$, percebemos que houve maior prevalência de baixos níveis (deficiência e insuficiência), isto é, vitamina $D$ sérica menor que $30 \mathrm{ng} / \mathrm{dL}$, no grupo de idosos portadores de manifestações musculoesqueléticas crônicas. Dessa forma, podemos inferir que haja associação entre níveis séricos reduzidos de vitamina $\mathrm{D}$ e o desencadeamento de dor crônica, além do desenvolvimento de sintomas depressivos e de depressão.

\section{REFERÊNCIAS}

1. ABDUL-RAZZAK KK, et al. Vitamin D Is a Potential Antidepressant in psychiatric Outpatients. Int. Journal of Clinical Pharmacology and Therapeutics, 2018, 56(12): 585-596.

2. ABDUL-RAZZAK KK, et al. Vitamin $D$ as Potential Antidepressant in Outpatients with Musculoskeletal Pain. Int. Journal of Clinical Pharmacology and Therapeutics, 2018,4 v. 56, p. 400-410, 2018.

3. BELFER I. Pain in women. Agri, 2017, 29(2): 51-54.

4. CAI C. Treating Vitamin D Deficiency and Insufficiency in Chronic Neck and Back Pain and Muscle Spasm: A Case Series. The Permanente journal, 2019, 23: 8-10.

5. CORSI N, et al. Prevalence, characteristics and treatment of chronic pain in elderly patients hospitalized in internal medicine wards. European Journal of Internal Medicine, 2018, 55: 35-39.

6. EDWARDS RR, et al. The Role of Psychosocial Processes in the Development and Maintenance of Chronic Pain. Journal of Pain, 2016, 17(9):70-92.

7. FERRETTI F, et al. Quality of life in the elderly with and without chronic pain. Brazilian Journal Of Pain, 2018, 1(2): 111-115.

8. HELDE-FRANKLING M, et al. Vitamin D in pain management. International Journal of Molecular Sciences, 2017, 18(10): 1-9.

9. HOLICK MF, et al. Evaluation, treatment, and prevention of vitamin D deficiency: An endocrine society clinical practice guideline. Journal of Clinical Endocrinology and Metabolism, 2011, 96(7): 1911-1930.

10. ISHAK WW, et al. Pain and Depression: A Systematic Review. Harvard Review of Psychiatry, 2018, 26(6): 352-363.

11. LARSSON C, et al. Chronic pain in older adults: prevalence, incidence, and risk factors. Scandinavian Journal of Rheumatology, 2017, 46(4): 317-325.

12. MANSON JE. Pain: Sex differences and implications for treatment. Metabolism: Clinical and Experimental, 2010,59:1620.

13. MCCABE PS, et al. Low vitamin D and the risk of developing chronic widespread pain: Results from the European male ageing study. BMC Musculoskeletal Disorders, 2016, 17(1): 1-9.

14. MIRANDA D, et al. O envelhecimento populacional brasileiro- desafios e consequências. Revista Brasileira de Geriatria e Gerontologia, 2016, 19(3): 507-519.

15. SHENG J, et al. The Link between Depression and Chronic Pain: Neural Mechanisms in the Brain. Neural Plasticity, 2017.

16. SORGE RE, et al. Sex Differences in Pain. Journal of Neuroscience Research, 2017, 95(6): 1271-1281.

17. TANG NK, et al. Suicidality in chronic pain: a review of the prevalence, risk factors and psychological, 2006 apud RACINE, M. Chronic pain and suicide risk: A comprehensive review, 2018.

18. TUFIK S, et al. The interfaces between vitamin D, sleep and pain. Journal of Endocrinology, 2017, 234(1): 23-36.

19. WU Z, et al. The association between Vitamin D concentration and pain: A systematic review and meta-analysis. Victorian Literature and Culture, 2018, 21(11): 2022-2037.

20. YANG CW, et al. Screening tools for neuropathic pain. Journal of the Chinese Medical Association, 2018, 81(1): 1-3. 